\title{
Research Progress on Synchronous Control of Mechanical System
}

\author{
Hui Chen ${ }^{1,}$, Zhimin Qiu² \\ ${ }^{1,2}$ Nanchang Institute of Science and Technology, 330108 \\ achenhuijx@126.com
}

Keywords: mechanical system; synchronous control; research progress

\begin{abstract}
With the continuous development of economic and society, there is a new momentum in the development of modern manufacturing, marked by more complicated production tasks and high accuracy production requirements. Many times in order to complete the complex production task, we need to put multiple mechanical equipment and parts into operation at the same time, which puts forward new requirements for synchronous control operation of mechanical system. Under the new manufacturing development situation, synchronous control method of mechanical system has been developed constantly. This paper carries out the related analysis and inquiry on synchronous control of mechanical system .
\end{abstract}

Under the new situation, the scale of manufacturing has been expanded. The large-scale manufacturing tasks require multiple collaborative work to complete, which puts forward higher requirements for collaborative means and methods of mechanical system. In order to provide reliable technical support for the modern manufacturing production, various related research units and good production units carried the related research on mechanical system synchronous control method, which obtained the related research results and promoted the development of modern manufacturing industry.

\section{Research on synchronous control method of multiple machinery}

The synchronous control method of more than one machinery has been widely applied in the manufacturing process of the automobiles, complicated equipment, large equipment. Synchronous control method of more than one machinery simply refers to the synchronous control method of the application of multiple mechanical arm. In this process, the application of multiple mechanical all at once concerted multiple motor performance. The traditional coordination work need to measure internal force, which has large limitations in the process of practical application. Nevertheless, the application of synchronous control method in multiple mechanical arm can effectively solve the problem without measuring internal force, which represents a important research progress on synchronous control method of mechanical system in recent years. As a typical nonlinear strong coupling system, mechanical arm system faces great difficulty in being controlled synchronously. Especially in the uncertain system and external disturbance, it is more difficult to design a synchronous control system of multiple mechanical arm. Joint space synchronous control and task space synchronous control are two main algorithms, but their methods to obtain knowledge are different. The joint space synchronous control method is a hot-spot of current research, and its results is relatively abundant. But with the continuous development of machinery industry, a lot more work tasks of mechanical arm has been widely used in the task space, and the synchronous movement of Joint does not guarantee that the synchronous movement of task space. So the synchronous control of task space has become a research focus.

\section{Research on synchronous control of joint space}

Synchronous control of joint space means that the design of synchronous control method to be carried on multiple mechanical arm, which can let displacement and speed of joint related to each mechanical equipment can realize synchronous movement. The application of this kind of control method in synchronous control operation of mechanical system can accurately describe the 
relationship between the system movement. The application of synchronous control of Joint space in mechanical arm joint exercise can overcome the negative impact on joints synchronous movement caused by the uncertainty of system, the external environment disturbance. However, some problems and factors related to kinematics are not taken into consideration. Thus when uncertain problems emerge in kinematics, joint synchronous control method is not enable to play an important role in mechanical production process.

\section{Synchronous control research on the task space}

Synchronous control on the task space refers to control synchronous calculation of the method according to the position and speed executed by mechanical arm in planning trajectory. The utilization of synchronous control on the task space in multiple mechanical arm is closely linked with machinery production task, so it plays a more direct role in the mechanical manufacturing process. Compared with joint synchronous control methods, synchronous control method of the task space emphasizes the uncertainty of dynamics, but it didn't analyze the impact of external interference factors, there is a big difficulty in theory research.

\section{Synchronous control method research on parallel robot}

Compared with synchronous control method of other mechanical system, parallel robot is a relatively closed organization, with great rigidity, operation schedule and strong capacity of load and bearing, which plays an important role in the production process of current large manufacturing. In the process of practical application, the cooperative motion of parallel strut provides important technical support to ensure the parallel robot platform to track the desired trajectory. To some extent, the synchronous control algorithm of parallel robot can improve the quality of control performance based on platform tracking and the coordination of operation between each strut. At present, synchronous control method for parallel robot is studied by many scholars and research institutions at home and abroad. They have integrated the dynamic model, adaptive synchronous control method and the principle of synovial membrane with the application of strut cooperative movement closely. According to the characteristics of the parallel robot kinematics, a series of synchronized error concept has been put forward and a variety of different synchronous control algorithm has been designed. But kinematics relationship of parallel robot form is relatively complex, the synchronous control algorithm is also relatively complex. Therefore, how to design a simple, convenient and efficient synchronous control algorithm of parallel robot is the important direction and trend of synchronous control method of mechanical system now.

\section{Research on formation control method of mobile robots}

Formation with multiple vehicles and aircraft and in satellite attitude are the main forms of mobile robots formation, the synchronous control method of mechanical system has been widely used in unmanned aircraft manufacturing industry and mineral exploration, investigation and other related industries. The research of mobile robot formation control method is still in a relatively primary stage, which means the existing methods can only ensure mobile robots are operated by the set formation, the relevant movement process cannot be effectively under technical constraints. At present, the mobile robot formation control is one of research traits of the mechanical system synchronous control method. With the study at the initial stage of development, there is a large space in technology research and development.

\section{Control study on the consistency of multiple agents}

This study is mainly about the position, velocity of mechanical rigid particle, and the synchronous control of mechanical system is an important category of control study on the consistency of multiple agents. With the continuous development of smart technology, its application in the field of mechanical manufacturing get deeper. Control on the consistency of 
multiple agents is a hot-spot research field where a large number of papers published in this research field. Time-varying time delay, time varying topology is the research focus of control on the consistency of multiple agents. Multi-agent consistent control research develop rapidly, new research and use methods appear constantly, but these methods are mostly based on linear system design of mechanical system, cannot get effective application in nonlinear mechanical systems. But the present study methods and ideas of multi-agent consensus control research has certain enlightening role in mechanical system synchronous control related research, and a lot of research results can provide abundant theoretical support in the field of mechanical synchronous control research, for related researchers which worth studying and drawing lessons from it.

\section{Summary}

Mechanical system synchronous control method is developed with the continuous development of mechanical manufacturing. In recent years, with the continuous development of mechanical manufacturing, the research on synchronous control method of mechanical system has also achieve relevant research results. And many fields has made certain research results such as synchronous control method of multiple machinery, synchronous control method of the parallel robot and mobile robot formation control method. However, the development of synchronous control method of mechanical system still needs further exploration and development.

\section{Reference:}

[1] Gang Chen, Yunlong Yue, Qing Lin, Ming Yu. The design of coordinate controller on distributed multibody mechanical system[J]. Journal of University of Science and Technology of China, 2013, (1):29-34+41.

[2] Qian Ding, Hongmei Zhai. Research progress on friction dynamic of mechanical system[J]. Advances in mechanics, 2013, (1):112-131.

[3] Yadong Zhao, Yongrui Zhao, Baohua Cui, Lan Zhang. Research progress on synchronous control of mechanical system[J]. Journal of Shandong University of Science and Technology(Natural Science) ,2013,(3):1-6.

[4] Rao Hua, Dongya Zhao, Lianchun Long. Research on synchronously tracking control method of task space of multiple mechanical arm [A]. Beijing Society of Theoretical and Applied Mechanics. The collected papers on 19th academic annual conference of Beijing Society of Theoretical and Applied Mechanics[C]. Beijing Society of Theoretical and Applied Mechanics:,2013:2.

[5] Mingzhe Yu, Huali Wu, Jinghui Gao. Research progress on synchronous control of fractional-order chaotic system[J].Journal of Naval Aeronautical and Astronautical University, 2014, (6):535-542. 